\title{
Finding hope amidst a pandemic
}

\author{
Vasvi Singh, $M D^{\mathrm{a}}$ \\ a Cardiovascular Imaging Program, Cardiovascular Division and Department of Radiology, \\ Brigham and Women's Hospital and Harvard Medical School, Boston, MA
}

Received Apr 24, 2020; accepted Apr 30, 2020

doi: $10.1007 /$ s12350-020-02183-4

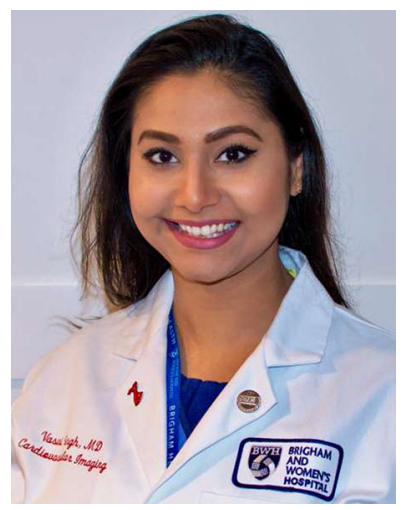

\section{SUMMARY}

The COVID-19 global pandemic has plundered all aspects of life. Healthcare professionals across the nation are under unprecedented pressure to ensure best patient care while balancing protection of their own health and that of their families. Healthcare systems are struggling to find solutions with limited resources. There is fear surrounding our very existence, and each day appears to bring worse news than the one before. So how do we, as a healthcare community find hope, or even optimism amidst this pandemic? Just as extraordinary medical discoveries have emerged from wartime medicine, many new innovations will happen during this crisis too.

The COVID-19 global pandemic has plundered all aspects of life as we know it. Healthcare professionals across the nation are under unprecedented pressure to

All editorial decisions for this article,including selection of reviewers and the final decision, were made byguest editor Saurabh Malhotra, MD, MPH.

Reprint requests: Vasvi Singh, MD, Cardiovascular Imaging Program, Cardiovascular Division and Department of Radiology, Brigham and Women's Hospital and Harvard Medical School, 75 Francis St, Boston, MA 02115; vsingh7@bwh.harvard.edu

J Nucl Cardiol 2020;27:1637-9.

$1071-3581 / \$ 34.00$

Copyright (C) 2020 American Society of Nuclear Cardiology. ensure the best patient care while balancing protection of their own health and that of their families. Healthcare systems are struggling to find innovative solutions with limited resources. There is fear, anguish, and ambiguity surrounding our very existence, and each day appears to bring worse news than the one before. So how do we, as a healthcare community find hope, or even optimism amidst this pandemic?

Just as extraordinary medical discoveries have emerged from wartime medicine, where battlefield medical personnel were compelled to innovate in the face of mass misery, many new discoveries will happen during this crisis too. The Centers for Medicare and Medicaid Services issued a first time series of temporary regulatory waivers and rule amendments towards providing the US healthcare system with greater flexibility to care for patients during the COVID-19 pandemic (Figure 1). Amongst chief actions were Medicare coverage for telehealth visits, virtual check-ins and e-visits; and waiving penalties for HIPAA violations when healthcare personnel use more accessible technologies such as Skype or FaceTime for patient interaction. Many new medical services have been added to Medicare's telehealth list, which are available not only to those residing in rural areas or are affected by COVID-19, but to all patients in need. Provisions like drive-up testing and availability of technicians to go to patients' homes, is invaluable for those with limited mobility, lack of social support, means of transportation and distant healthcare facilities. Expanded home monitoring ability with appropriate reimbursement increases outreach to patients, and ensures prompt healthcare delivery for all. Reducing administrative red tape and elimination of unverified quality metrics in healthcare policies ensures facilitated access to care in every patient setting, including homes. Allowing patients, a tele access to generalists and subspecialty physicians eliminates barriers of physical presence that encourages patients to seek timely medical attention, and improves patient compliance to appointments, follow-ups and thereby therapy. 


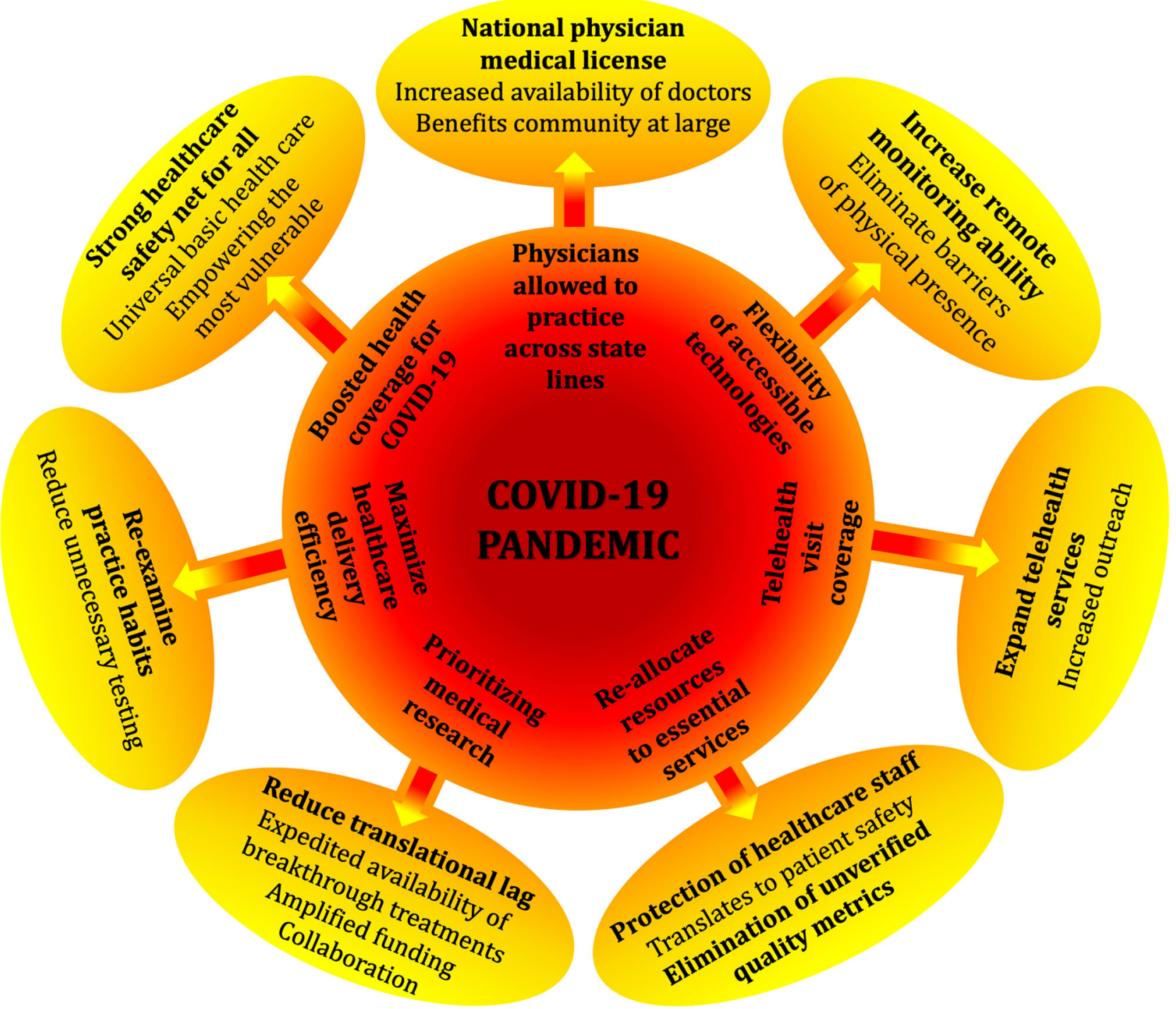

Figure 1. Finding rays of hope: unique opportunities to advance healthcare reforms beyond the pandemic.

Physicians licensed in one state have now been allowed to provide care across state lines to meet increasing demands. In the USA, an entangled web of national and state regulations controls physician licensing. Although all physicians in the USA, irrespective of their state of residence or training pass either the United States Medical Licensure Examinations or the Comprehensive Osteopathic Medical Licensing Examination, then receive medical training following national guidelines set by the Accreditation Council for Graduate Medical Education, however, to finally practice medicine, they are required to obtain and maintain a separate license for each State with its exclusive patchwork of requirements. How does that make any sense? This split system makes it arduous for physicians to care for patients in other states, and in particular obstructs telemedicine, a fact that was highlighted by this pandemic. The application process and requisites for each state are varied, burdensome, impose a high fee that is usually several hundred dollars, and state agencies typically take months to process these long applications, thereby discouraging physicians from practicing across state lines. These redundant and fragmented licensure practices create excessive administrative burdens, impose direct costs on physicians who must decipher and comply with multiple states' licensure requirements, and places indirect high costs to patients and hospitals by preventing physicians from practicing in those 
locations where the greatest need exists. For example, there is a pervasive shortage of specialists in rural America and patients are often forced to commute long distances and endure lengthy wait times to be seen by a physician. These barriers to access of health care, ultimately contribute to worse population health outcomes and increase healthcare burden. Therefore, the need for physicians without state borders exists independent of the pandemic. The expanded coverage of telehealth services and ability to practice medicine across state confines when made permanent, would continue to benefit the community at large, even beyond this public health crisis.

The COVID-19 pandemic has obligated us all to take a step back, analyze and re-allocate resources to the most essential services that revolve around patient care, such as protection of healthcare staff that congruently translates to patient safety. It also provides us with an opportunity to re-examine our practice habits that would maximize efficiency of healthcare delivery, such as reduction in unnecessary testing that in part is motivated by medicolegal implications. Through shifting focus to what impacts change in patient management the most, we would be able to eliminate the tradition of local redundant practices while progressing towards patientcentered care, rather than system-centered care. Several medical society statements have been expeditiously published to collectively guide adaptations in clinical care and safety measures to ensure best practices in the COVID-19 era.

While the country edgily waits for a vaccine and a miraculous cure, this critical time in history calls attention to the importance of prioritizing medical research and innovation, through amplified central and regional funding. For years, biomedical scientists have been coping with the tension between their aspirations to conduct innovative clinical research and the burdensome regulations that hold up timely progression of their research from the bench to the bedside. The average translational lag between a medical breakthrough and the first highly cited study demonstrating its clinical utility, is several years. Each regulation while established in good intent, when combined with several others creates a trap challenging to navigate even for the experienced researcher. Sadly, the manner in which regulations have piled on, it has discouraged a large number of highly-skilled multi-tasking clinicians to perform research as the system is too cumbersome. The regulators need to establish a more dynamic system that lowers bureaucratic hurdles and effectively spots problems to ensure patient safety, without stifling academic medical research and obstructing patient access to innovative treatment. Reducing the administrative hurdles and allowing collaborative medical research to be conducted and published rapidly would allow expedited availability of breakthrough treatments.

It has been said that when I is replaced with We, Illness becomes Wellness. This is definitely the living truth for many low-income families and elderly individuals who are worst hit by the COVID-19 pandemic. With lack of healthcare insurance, reduced economic mobility, and the high cost of medical care in the USA, this pandemic has uncovered the fragile healthcare safety net that leaves the most vulnerable communities to bear a large brunt of the disease. Marginal groups segregated principally by race, finances and immigration status are more likely to be exposed to the virus. In addition, medical research has shown that low income is associated with higher prevalence of chronic health conditions such as cardiovascular disease and diabetes, co-morbidities that increase morbidity and mortality with COVID-19 infection. Majority of small business employees presently do not receive health insurance. While the government has currently increased financial coverage for COVID-19 testing, prevention and treatment, there is a dire need beyond the pandemic for a nationwide plan to provide basic health care access to all. Free preventive care services for cardiovascular disease, diabetes, depression, and other health challenges, immunization schemes and wellness visits would empower the minority communities to proactively prevent and manage chronic health conditions. This would in turn reduce the overall mounting cost of health care, both for the individual patient and for healthcare systems.

This pandemic gives us the unique opportunity to understand the menacing limitations of the current processes, exposes the regulations that in fact serve as barriers for effective healthcare delivery, and at the same time provides simplistic solutions on how to overcome them (Figure 1). The desperation of this pandemic brings us all together towards creating a better future. As the famous Bostonian philosopher said, "Every sunset brings the promise of a new dawn".

Publisher's Note Springer Nature remains neutral with regard to jurisdictional claims in published maps and institutional affiliations. 